\title{
Spatial Filter Housing for Enhancement of the Shielding Effectiveness of Perforated Enclosures with Lossy Internal Coating: Broadband Characterization
}

\author{
Khalid F. A. Hussein \\ Microwave Engineering Department, Electronics Research Institute, Cairo 12622, Egypt \\ Correspondence should be addressed to Khalid F. A. Hussein; khalid_elgabaly@yahoo.com
}

Received 14 May 2012; Revised 16 September 2012; Accepted 30 November 2012

Academic Editor: Tamer Ibrahim

Copyright (C) 2013 Khalid F. A. Hussein. This is an open access article distributed under the Creative Commons Attribution License, which permits unrestricted use, distribution, and reproduction in any medium, provided the original work is properly cited.

\begin{abstract}
This work is concerned with studying and enhancement of the electromagnetic shielding effectiveness of open metallic enclosures with openings over a wide range of the frequency including the UHF band. The suggested methods depend on the suppression of the excessive power penetrating the enclosure cavity especially at its resonances by a variety of methods that include increasing aspect ratio of the rectangular aperture, splitting the opening into a number of apertures, coating the internal walls of the metallic enclosure with a multilayered lossy material of the appropriate conductivity profile, and, finally, placing the metallic enclosure inside a spatial filter housing. A minimum value of $20 \mathrm{~dB}$ is achieved for the shielding effectiveness using the suggested methods. The present work also provides a study to investigate the effect of the direction and polarization of the incident plane wave on the shielding effectiveness of the enclosure.
\end{abstract}

\section{Introduction}

Metallic shielding is one of the major means to prevent electronic equipment from electromagnetic interference in electromagnetic compatibility (EMC) design [1-3]. The shielding efficiency of a metallic enclosure is characterized by its shielding effectiveness (SE), which is defined as the ratio of the electromagnetic field without the presence of the enclosure to the field with the presence of the enclosure at the same observation point. However, the integrity of these enclosures is often compromised by apertures and slots used to accommodate visibility, ventilation, or access to interior components. Such openings allow exterior electric and magnetic fields to penetrate to the interior space, where they may couple onto printed circuit boards (PCBs) thus inducing currents and voltages on interior conductors. It is important to study the EM shielding effectiveness of shielding enclosures in the presence of these apertures.

Calculated shielding effectiveness depends on the position inside the enclosure where the fields are calculated. For walls of the enclosure that are considered to be perfectly conducting, the penetration of fields is only through the apertures. There is a variety of modeling techniques associated with EMC, such as Method of Moments (MoM) [46], Finite Element Method (FEM) [7], and Finite-difference Time-domain method (FDTD) $[8,9]$. Different modeling techniques are suited to different problems. In the present work, the FDTD technique is preferable, since it is an effective time-domain volume-based method.

The maximum penetration of the electromagnetic power occurs when the plane wave is normally incident on the aperture plane with the electric field parallel to the same plane and normal to the longer side of the rectangular aperture. Of course, this is the worst case regarding electromagnetic shielding requirements. Therefore, the determination of the shielding effectiveness is usually achieved by considering a plane wave at a normal angle of incidence. Nevertheless, the effect of considering other angles of incidence and polarization has been proven to be significant [4].

Improvement of the shield effectiveness of the rectangular enclosure at the cavity resonant frequency [6] can be obtained by using lossy internal coating to the enclosure walls, thus 


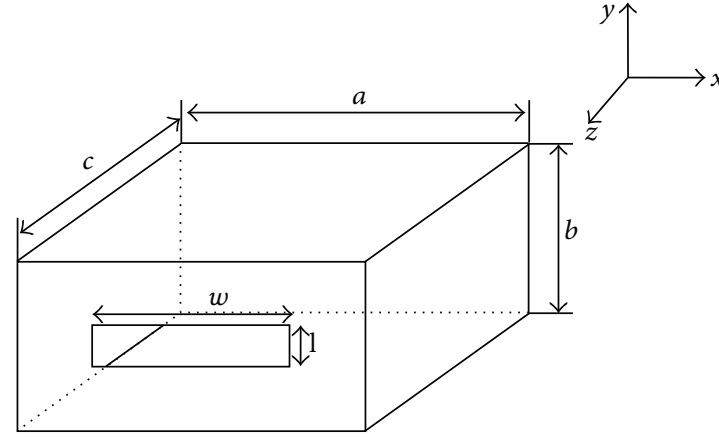

(a) Single apertures

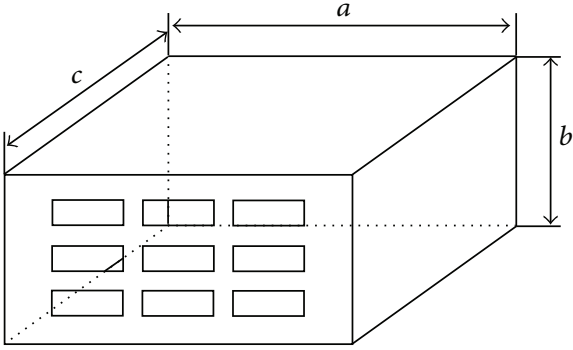

(b) Multiple apertures

FIGURE 1: Geometry of a rectangular metallic enclosure with rectangular apertures.

absorbing some of the internal fields, which leads to the reduction of the $Q$-factor of the cavity and, hence, reducing its ability to store electromagnetic energy.

Additional improvement of the shielding effectiveness is achieved in the present work by placing a planar band-stop spatial filter in front of the aperture such that most of the incident power is filtered out before penetrating the cavity. Of course the spatial filter is designed so that its stop band is centered on the cavity resonant frequency.

Another suggestion to improve the shielding effectiveness of such an enclosure is by splitting the single aperture to multiple apertures arranged in rows and columns such that the total area of the multiple apertures is the same as that of the original one. Splitting an aperture into multiple smaller apertures has the effect of decreasing the coupling between the exterior and interior of the enclosure cavity due to the additional vertical and horizontal metallic strips between the small apertures.

The above three approaches for increasing the shielding effectiveness are combined together, and the resulting improvement in the shielding effectiveness of the enclosure is investigated over a wide frequency range.

\section{Shield Design}

A rectangular metallic enclosure with a single or multiple apertures in one of its side walls is shown in Figure 1. The enclosure has the dimensions $a \times b \times c$. The rectangular aperture has the dimensions $l \times w$.

Like all cavities, the interior of a conducting rectangular box has discrete resonance modes. These internal modes are obtained as the solutions of source-free Maxwell's equations in the region bounded by the walls of the rectangular box. These modes can exist in an empty rectangular cavity if the largest cavity dimension is greater than or equal to one half a free space wavelength. Below this cutoff frequency, the cavity resonance cannot exist.

The resonant frequencies can be calculated for a rectangular box-shaped enclosure using the following expression [7]:

$$
F_{(m, n, p)}=\frac{c_{o}}{2} \sqrt{\left(\frac{m}{a}\right)^{2}+\left(\frac{n}{b}\right)^{2}+\left(\frac{p}{c}\right)^{2}},
$$

where $c_{o}$ is the speed of EM waves in free space and $m, n$, and $p$ represent the mode integers (one of which can be zero). It is obvious that the frequency of a resonance mode is inversely proportional to the related enclosure dimension. For the present case, the rectangular enclosure is designed such that its smallest dimension is that along the $y$-coordinate, so the resonance modes of the lowest frequencies are those modes with $n=0$, and, hence, the resonance mode of the lowest frequency is the $\mathrm{TE}_{101}$ mode.

To improve the shielding effectiveness of such an open metallic rectangular box it is suggested to modify its design by (1) increasing the aspect ratio of the rectangular aperture, (2) splitting the aperture into small-sized multiple apertures whose total area is the same as the original aperture, (3) using multilayered lossy coating on the inner walls of the rectangular box, and (4) placing an external band-stop spatial filter in front of the aperture. The evaluation of the proposed design will be discussed in detail in the remaining of the paper.

\section{Evaluation of the Shielding Effectiveness Using FDTD}

The shielding effectiveness is defined as

$$
\mathrm{EFS}=-20 \log \left(\frac{\left|E^{t}\right|}{\left|E^{i}\right|}\right)(\mathrm{dB}),
$$

where $E^{t}$ refers to the total electric field computed at the calculation or measurement point inside the cavity and $E^{i}$ refers to the incident electric field computed at the same location in the absence of the enclosure.

The metallic enclosure is subjected to an incident plane wave. The direction of incidence and polarization of the plane wave are as indicated in Figure 2.

An incident plane wave has its direction of propagation determined by the angles $\theta_{i}$ and $\phi_{i}$ as shown in Figure 2 . Another angle, $\psi_{i}$, is the angle between the projection of the electric field vector on the $x-y$ plane and the $x$-axis. Thus, knowing the direction of propagation, the angle $\psi_{i}$ determines the direction of the electric field, and hence it is called the polarization angle. 


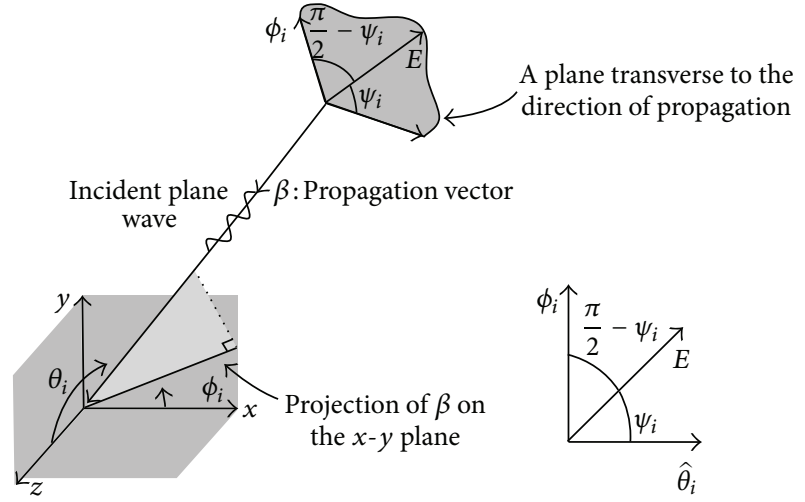

FIGURE 2: Definition of angle of incidence and polarization of the incident plane wave.

The FDTD method [10-12] is applied with an absorbing boundary condition to truncate the solution space whilst simulating free space. A computer memory efficient formulation of the split-field perfectly matched layer (PML) proposed by Berenger [13] is utilized throughout the present work. The metallic walls are considered as perfectly electric conductor (PEC) by enforcing the parallel electric field components to vanish on these walls.

The total field/scattered field (TF/SF) formulation [11] is utilized to calculate the fields inside the enclosure and, hence, the shielding effectiveness. Two forms of the plane waves are used: the first is a sinusoidal (continuous) plane wave, which is utilized to calculate the shielding effectiveness at a single frequency. The other plane wave is Gaussian pulsed and is used to calculate the shielding effectiveness over a specific frequency range. The detailed expressions for the continuous and Gaussian-pulsed plane wave are given as follows.

3.1. Continuous Plane Wave. According to the above description of the plane wave, one can write the following expressions for the incident fields of a continuous (sinusoidal) plane wave:

$$
\begin{aligned}
E_{x}^{i}= & E_{o}^{i}\left(\cos \psi_{i} \cos \theta_{i} \cos \phi_{i}-\sin \psi_{i} \sin \phi_{i}\right) \\
& \times e^{-j\left(\beta_{x} x+\beta_{y} y+\beta_{z} z\right)} e^{j \omega t}, \\
E_{y}^{i}= & E_{o}^{i}\left(\cos \psi_{i} \cos \theta_{i} \sin \phi_{i}+\sin \psi_{i} \cos \phi_{i}\right) \\
& \times e^{-j\left(\beta_{x} x+\beta_{y} y+\beta_{z} z\right)} e^{j \omega t}, \\
E_{z}^{i}= & -E_{o}^{i} \cos \psi_{i} \sin \theta_{i} e^{-j\left(\beta_{x} x+\beta_{y} y+\beta_{z} z\right)} e^{j \omega t},
\end{aligned}
$$

where $E_{o}^{i}$ is the amplitude of the incident electric field, $\beta_{x}$, $\beta_{y}$, and $\beta_{z}$ are the wavenumbers in $x, y$, and $z$ directions, respectively, and are given as $\beta_{x}=\beta \sin \theta_{i} \cos \phi_{i}, \beta_{y}=$ $\beta \sin \theta_{i} \sin \phi_{i}$, and $\beta_{z}=\beta \cos \theta_{i}$, and $\beta$ is the total wavenumber in free space. One can note that $\beta=\sqrt{\beta_{x}^{2}+\beta_{y}^{2}+\beta_{z}^{2}}$.
3.2. Gaussian-Pulsed Plane Wave. For a Gaussian-pulsed plane wave, the incident fields can be written as follows:

$$
\begin{aligned}
E_{x}^{i}= & \frac{4}{T \sqrt{\pi}} E_{o}^{i}\left(\cos \psi_{i} \cos \theta_{i} \cos \phi_{i}-\sin \psi_{i} \sin \phi_{i}\right) \\
& \times e^{-16 / c^{2} T^{2}\left[c\left(t-t_{o}\right)-(1 / \beta)\left(\beta_{x} x+\beta_{y} y+\beta_{z} z\right)\right]^{2}}, \\
E_{y}^{i}= & \frac{4}{T \sqrt{\pi}} E_{o}^{i}\left(\cos \psi_{i} \cos \theta_{i} \sin \phi_{i}+\sin \psi_{i} \cos \phi_{i}\right) \\
& \times e^{-16 / c^{2} T^{2}\left[c\left(t-t_{o}\right)-(1 / \beta)\left(\beta_{x} x+\beta_{y} y+\beta_{z} z\right)\right]^{2}}, \\
E_{z}^{i}= & -\frac{4}{T \sqrt{\pi}} E_{o}^{i} \cos \psi_{i} \sin \theta_{i} \\
& \times e^{-16 / c^{2} T^{2}\left[c\left(t-t_{o}\right)-(1 / \beta)\left(\beta_{x} x+\beta_{y} y+\beta_{z} z\right)\right]^{2}},
\end{aligned}
$$

where the time period $T$ determines the Gaussian pulse width and $t_{o}$ is the time at which the Gaussian pulse reaches its maximum value.

\section{Results and Discussions}

The following presentations and discussions are concerned with the frequency response of the shielding effectiveness of a rectangular box enclosure with single or multiple rectangular apertures as well as the improvement of this response by different methods. Some of the results are concerned with the dependence of the shielding effectiveness of such enclosures on the size and the aspect ratio of the aperture in case of an enclosure with a single aperture. Other results concerned with the effect of the arrangement and number of apertures in the case of an enclosure with multiple apertures are presented and discussed. The other parameters affecting the shielding effectiveness such as the direction of incidence and the polarization of the incident plane wave are discussed, and the related results are presented.

The results concerned with the improvement of the shielding effectiveness using lossy coating on the inner walls of the enclosure and/or a spatial band-stop filter which is placed in front of the aperture are presented and discussed.

It may be worth noting that the results presented in this section are obtained by applying the FDTD method as described above through a computer program developed using $\mathrm{C}++$ language. For the sake of investigating the validity of the developed program, some of the results obtained here are compared with other published ones.

\subsection{Characterization of the Shielding Effectiveness of a Rectan- gular Enclosure with a Rectangular Aperture}

4.1.1. Degradation of the Shielding Effectiveness of an Enclosure with a Single Aperture at Internal Resonance. Consider a 30 $\times 12 \times 30 \mathrm{~cm}^{3}$ enclosure with a single aperture of dimension $20 \times 3 \mathrm{~cm}^{2}$. This enclosure is illuminated by a normally incident plane wave of $y$-polarized electric field. The shielding effectiveness is obtained by calculating the electric field at the 


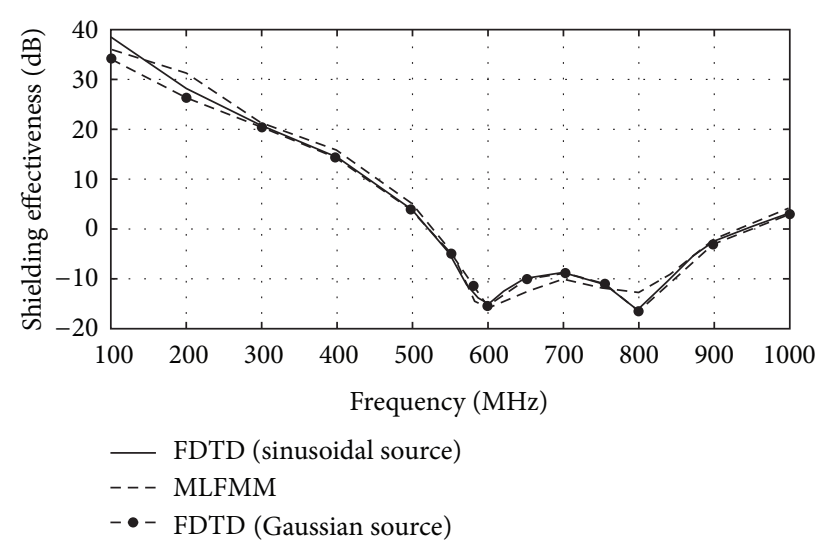

FIGURE 3: Comparison among the values of the SE obtained using three different methods for a box-shaped enclosure of dimensions 30 $\times 12 \times 30 \mathrm{~cm}^{3}$ with rectangular aperture of dimensions $20 \times 3 \mathrm{~cm}^{2}$ illuminated with $E_{y}$-polarized plane wave.

center of the enclosure over the frequency range of $100 \mathrm{MHz}$ to $1 \mathrm{GHz}$. The FDTD method is applied for this purpose by dividing the three dimensional space including the metallic enclosure into $180 \times 180 \times 180$ cubic cells each of dimensions $0.5 \times 0.5 \times 0.5 \mathrm{~cm}$. The enclosure wall thickness is one cell of perfect conductivity. The total/scattered field formulation is used with a perfectly matched layer (PML) of 8-cell thickness as absorbing boundary conditions.

Gaussian-pulsed plane wave is applied, and the discrete Fourier transform is applied. The results are shown in Figure 3 and compared with those obtained using the multilevel fast multiple moment method (MLFMM) [14]. As shown in the figure, there is a good agreement between the results, which insures the validity of the applied FDTD algorithm.

More comparisons can be achieved among the results concerning the same enclosure with an aperture of dimensions $30 \times 12 \mathrm{~cm}^{2}$ obtained using different methods. The SE is calculated over the frequency range of interest and compared with that obtained in [5]. The comparison is presented in Figure 4 showing good agreement among the results.

\subsubsection{Effect of the Aperture Width on the Shielding Effectiveness} of an Enclosure with a Single Aperture. It may be important to investigate the effect of the aperture width (horizontal dimension) on the SE of the enclosure while keeping the aperture vertical dimension unchanged. One may expect that by decreasing the aperture width the SE increases as the area through which the electromagnetic power can penetrate into the enclosure cavity is reduced. Figure 5 shows a plot for the SE against the frequency with varying the aperture width from 5 to $30 \mathrm{~cm}$ while keeping the aperture height at $10 \mathrm{~cm}$. An $E_{y}$-polarized plane wave is assumed to be normally incident on the aperture. As shown in Figure 5, the SE decreases rapidly when the frequency approaches the internal resonance of the cavity. Two resonances appear in the figure: the first one is related to the resonant mode $\mathrm{TE}_{101}$ (at about $707 \mathrm{MHz}$ for the closed cavity) and the second is related to $\mathrm{TE}_{102}$ mode (at about $1118 \mathrm{MHz}$ for the closed cavity). For

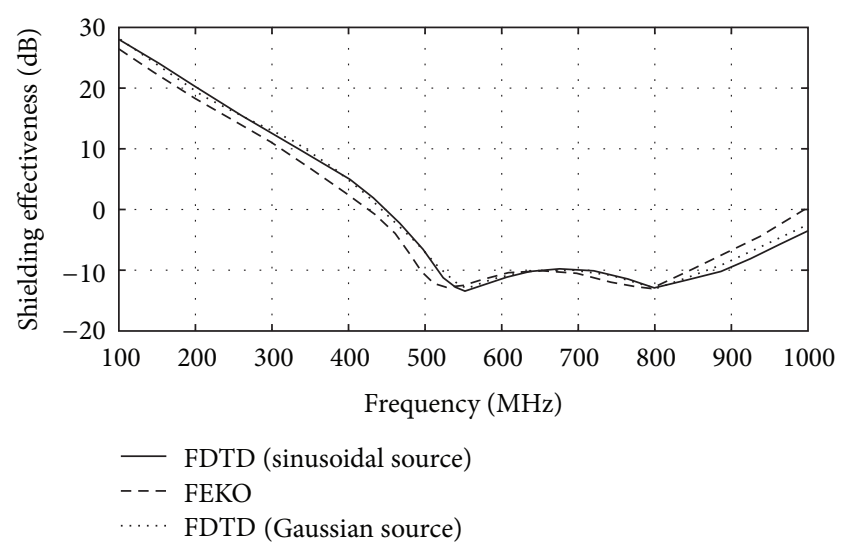

FIgURE 4: Comparison among the values of the SE obtained using three different methods for a box-shaped enclosure of dimensions 30 $\times 12 \times 30 \mathrm{~cm}^{3}$ with rectangular aperture of dimensions $30 \times 12 \mathrm{~cm}^{2}$ illuminated with $E_{y}$-polarized plane wave.

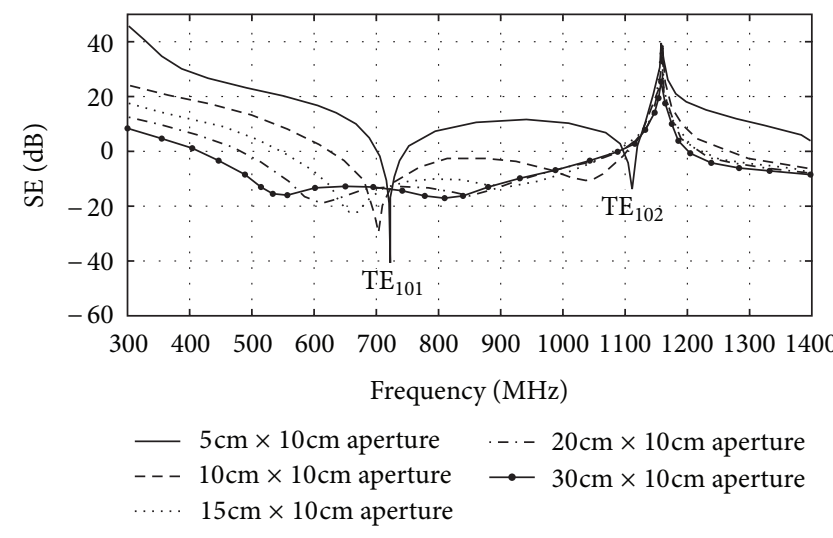

FIGURE 5: Shielding effectiveness versus frequency at the center of a box-shaped enclosure of dimensions $\left(30 \times 12 \times 30 \mathrm{~cm}^{3}\right)$ with rectangular aperture of different dimensions.

an open cavity the resonant frequencies for internal modes decrease with increasing the aperture width; this is noticeable in the SE/frequency plot of Figure 5.

It is shown in the figure that as the aperture gets narrower, the SE shows very sharp minimum of high amplitude at the $\mathrm{TE}_{101}$ resonant mode. As the aperture is getting wider, the minimum of the $\mathrm{SE}$ at $\mathrm{TE}_{101}$ becomes less sharp, has lower amplitude, and occurs at a lower frequency.

4.1.3. Effect of Direction of Incidence of the Plane Wave on the Shielding Effectiveness of an Enclosure with a Single Aperture. The shielding effectiveness of a conducting rectangular box with a rectangular aperture depends on the direction of propagation of the incident plane wave. Figures 6 and 7 show the dependence of the shielding effectiveness on the elevation angle of incidence $\theta_{i}$ at different frequencies. According to the coordinate system shown in Figure 2 the plane wave is normally incident on the aperture plane when $\theta_{i}=0$; 


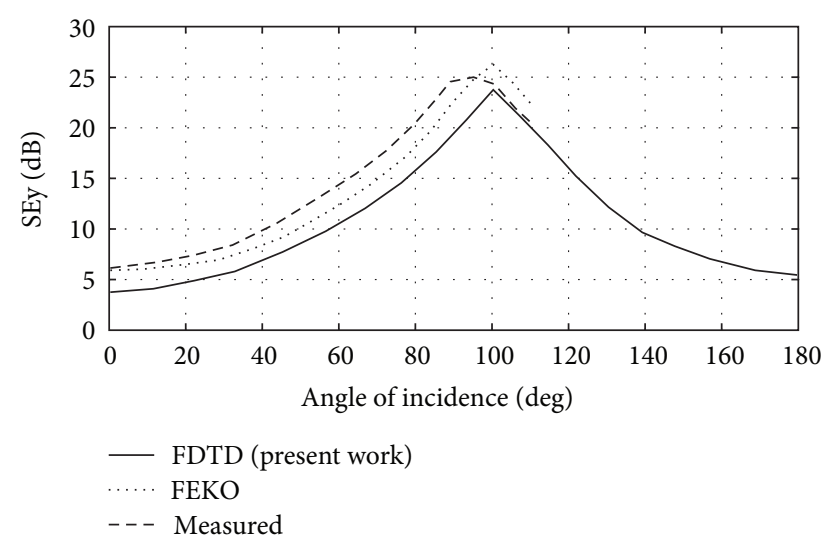

FIGURE 6: Shielding effectiveness of a box-shaped enclosure $(30 \times$ $\left.12 \times 30 \mathrm{~cm}^{3}\right)$ with a rectangular aperture $\left(20 \times 3 \mathrm{~cm}^{2}\right)$ against the elevation angle of incidence of the plane wave $\left(\theta_{i}\right), f=500 \mathrm{MHz}$.

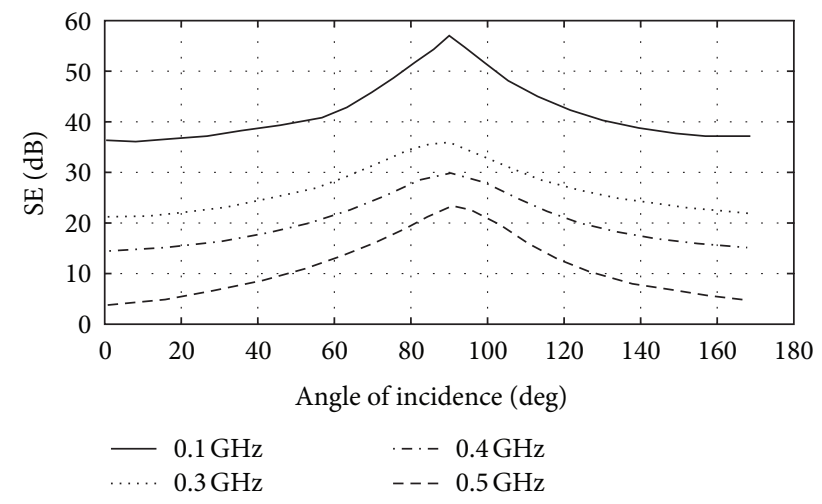

FIGURE 7: Shielding effectiveness of a box-shaped enclosure $(30 \times$ $\left.12 \times 30 \mathrm{~cm}^{3}\right)$ with a rectangular aperture $\left(20 \times 3 \mathrm{~cm}^{2}\right)$ against the elevation angle of incidence of the plane wave $\left(\theta_{i}\right)$ for different frequencies.

this gives the lowest value of the shielding effectiveness. The maximum value of the shielding effectiveness is obtained when the plane wave direction is parallel to the aperture plane $\left(\theta_{i}=90^{\circ}\right)$. It is clear, in Figures 6 and 7 , that the shielding effectiveness obtained when the plane wave is incident on the back of the shielding enclosure $\left(\theta_{i}=180^{\circ}\right)$ is slightly larger than that obtained when the plane wave is incident directly on the aperture face $\left(\theta_{i}=0^{\circ}\right)$. In Figure 6 , the results obtained in the present work are compared to those obtained in [5] using the commercial software package FEKO and those obtained through measurements. The comparison shows good agreement.

Figure 7 shows the dependence of the shielding effectiveness on the elevation angle of incidence $\theta_{i}$ for different values of the operating frequency. The results ensure that, at all the frequencies, the minimum shielding effectiveness is obtained when the plane wave direction is normal to the aperture plane, whereas the maximum shielding effectiveness is obtained when the plane wave direction is parallel to the aperture plane.

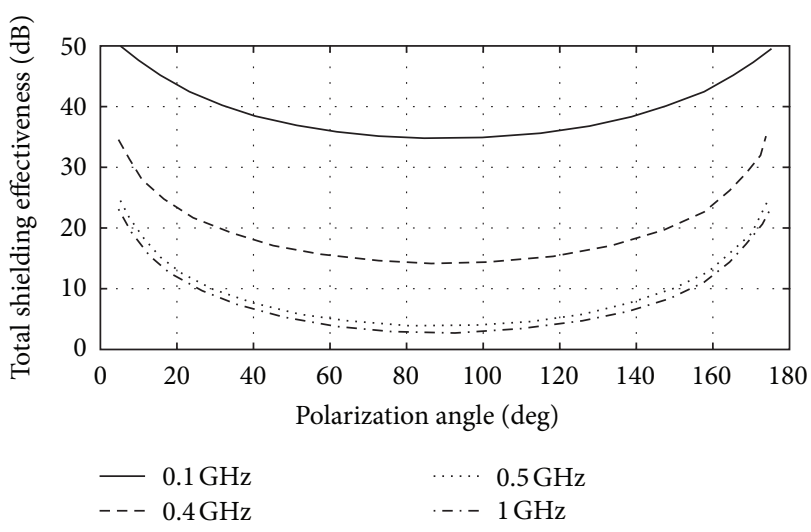

FIGURE 8: Shielding effectiveness versus polarization angle $\left(\psi_{i}\right)$ of the incident plane wave for a $30 \times 12 \times 30 \mathrm{~cm}^{3}$ box-shaped enclosure with $20 \times 3 \mathrm{~cm}^{2}$ aperture at different frequencies.

4.1.4. Effect of Polarization of the Incident Plane Wave on the Shielding Effectiveness of an Enclosure with a Single Aperture. The orientation of the electric field of the incident plane wave affects the shield effectiveness of a box-shaped enclosure with a rectangular cavity. The results presented in Figure 8 demonstrate the dependence of the shielding effectiveness on the polarization angle $\psi_{i}$. As shown in the figure, the minimum value of the shielding effectiveness is obtained when the electric field is parallel to the larger edge of the slot, whereas the maximum value is obtained when the incident electric field is normal to this edge of the slot. In this example, the plane wave is normally incident on the slot plane, and hence the incident electric field lies in the slot plane.

4.2. Improvement of the Shielding Effectiveness of a Rectangular Enclosure with a Rectangular Aperture. In the following, a variety of methods are presented to improve the shielding effectiveness of a rectangular metallic box with an aperture. Some of these methods depend mainly on preventing most of the incident power from entering the cavity while the other methods depend on suppressing the power penetrating into the cavity.

4.2.1. Improvement of the Shielding Effectiveness of the Enclosure by Increasing the Aspect Ratio of the Aperture. Let us define the aspect ratio of the rectangular aperture as the ratio between the length of the vertical side and that of the horizontal side. According to the plot shown in Figure 9, the shielding effectiveness increases with increasing the aspect ratio except at the resonant frequency of the enclosed cavity.

For further investigations of the effect of the aspect ratio on the shielding effectiveness, Figure 10 shows a comparison among the values of the shielding effectiveness of an enclosure of a single slot for three different values of the aspect ratio. It is clear in the figure that the shield effectiveness increases with increasing the aspect ratio over the entire frequency range except at the frequency corresponding to the internal resonance. 


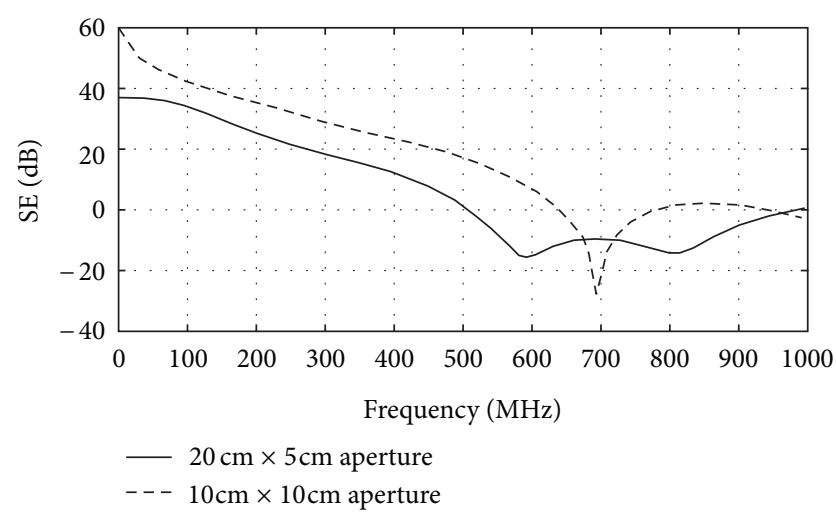

FIGURE 9: Shielding effectiveness versus frequency for a box-shaped enclosure of dimensions $30 \times 12 \times 30 \mathrm{~cm}^{3}$ with a single slot for different values of the aspect ratio and having the same area.

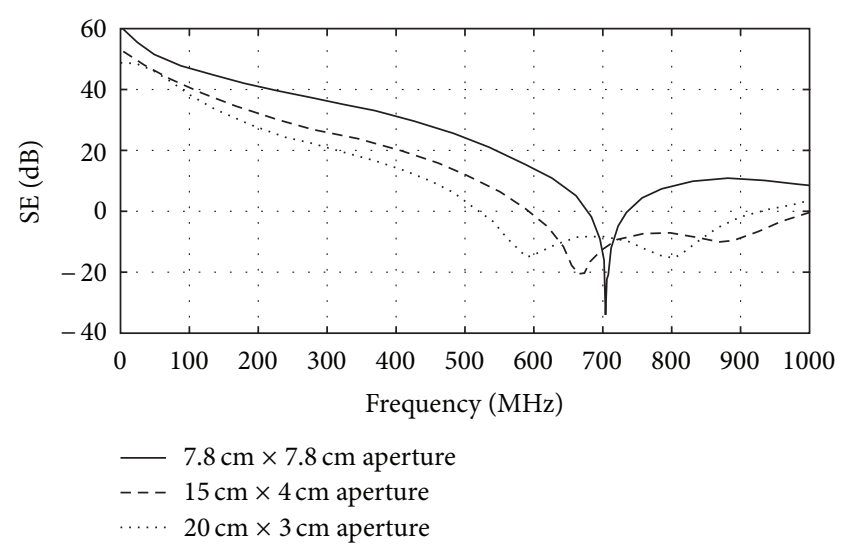

FIGURE 10: Shielding effectiveness versus frequency for a boxshaped enclosure of dimensions $30 \times 12 \times 30 \mathrm{~cm}^{3}$ with a single slot for different values of the aspect ratio and having the same area.

4.2.2. Improvement of the Shielding Effectiveness by Using Multiple Apertures instead of Single Aperture. If the single aperture on one of the rectangular enclosure is split into multiple apertures whose total area is the same as that of the original aperture, the shielding effectiveness may be improved. In other words, the single slot is replaced by a number of smaller slots arranged in a planner array as rows and columns. This leads to increase the shielding effectiveness of the shielding enclosure. Figure 11 shows a comparison between the shielding effectiveness of an enclosure with a single slot and that of two slots arranged in one row and two columns, where the two slots together have the same area of the single slot. It is shown in the figure that splitting the single slot into two adjacent slots results in increasing the shielding effectiveness except at the resonant frequency of the enclosure cavity. For further investigations of the effect of splitting the slot area into a larger number of smaller slots, Figure 12 shows a comparison among the shielding effectiveness of an

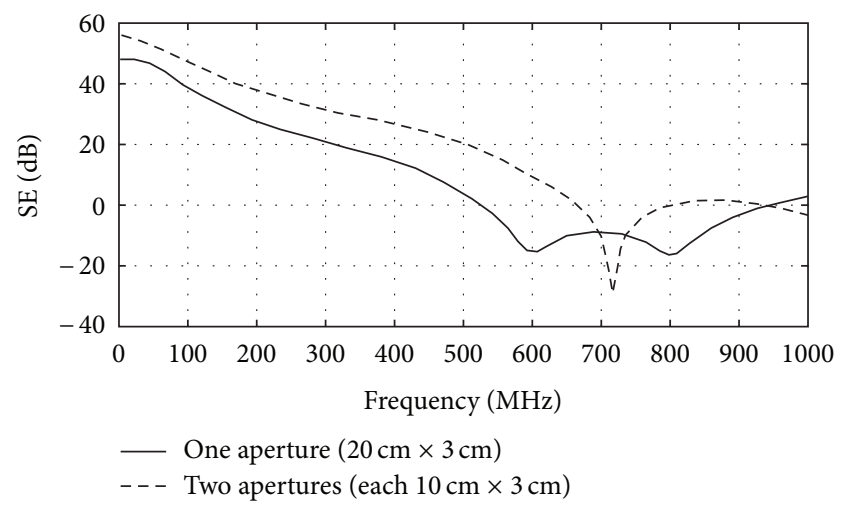

FIGURE 11: Shielding effectiveness versus frequency for a boxshaped enclosure of dimensions $30 \times 12 \times 30 \mathrm{~cm}^{3}$ with a single slot as that with two slots.

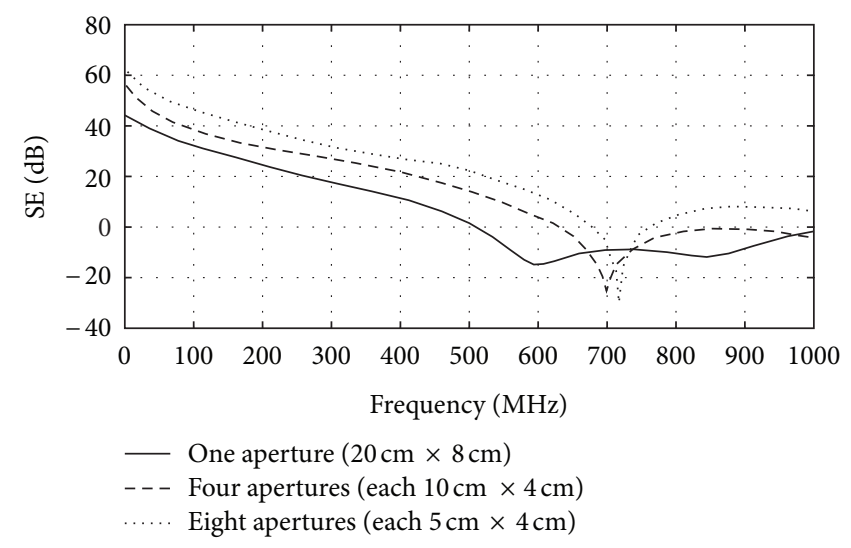

FIGURE 12: Shielding effectiveness versus frequency for a boxshaped enclosure of dimensions $30 \times 12 \times 30 \mathrm{~cm}^{3}$ with a different number of rectangular apertures.

enclosure of a single slot to that of an enclosure with four slots arranged as $2 \times 2$ array and that of an enclosure with eight slots arranged as $2 \times 4$ array. It is clear in the figure that as the number of slots increases, the shield effectiveness increases provided that the total area of the multiple slots is the same as that of the single slot. To ensure this result, a third case is presented in Figure 13 from which we can arrive at the same conclusion.

4.2.3. Improvement of the Shielding Effectiveness of Open Enclosures at Internal Resonance Using Lossy Coating on the Internal Walls. The electromagnetic power coupling from an incident wave to the interior of a rectangular cavity is enhanced at the internal resonances of the cavity. Consequently, the shielding effectiveness may be very low over narrow frequency bands around the internal resonance. The shield effectiveness of an open rectangular enclosure can be improved by diminishing or reducing the internal field at 


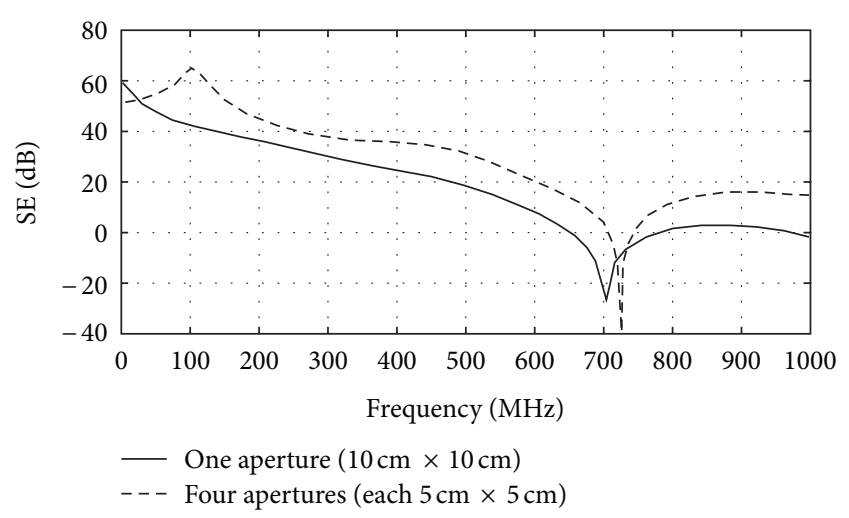

FIGURE 13: Shielding effectiveness versus frequency for a boxshaped enclosure of dimensions $30 \times 12 \times 30 \mathrm{~cm}^{3}$ with different number of rectangular apertures having the same total area.

such resonance. This can be achieved by coating the walls of the rectangular cavity by an absorbing material, which causes the internally generated fields to be partially absorbed into the wall coating, and hence the internal region of the enclosure seems to be surrounded by a free space region rather than a conducting boundary. In other words, the absorbing coating on the internal walls leads to reduce the Q-factor of the cavity and, hence, to reduce its ability to store electromagnetic energy. Figure 14 shows a comparison between the shielding effectiveness of an open box-shaped enclosure with an internal absorbing coating and that of the same enclosure without such coating. The results show that the shielding effectiveness is improved at the internal resonances corresponding to the $\mathrm{TE}_{101}$ and $\mathrm{TE}_{102}$ modes of the rectangular cavity; however, a slight reduction of the shielding effectiveness occurs over the low frequency range. This can be attributed to that the lossy coating on the internal wall has better absorption of the excessive electromagnetic power at the higher frequencies than its absorption at lower frequencies. On the other hand, the thickness of the coating layer results practically in reducing the inner dimensions of the enclosure cavity leading to a bad effect on the shielding effectiveness at lower frequencies.

It should be noted that to get a good absorbing coating, it is constructed up from several layers that achieve a specific conductivity profile. The improvement obtained in the case presented in Figure 14 is achieved using conductivity profile: $0.01,0.0316,0.1,0.316,1$, and $3.16 \mathrm{ohm}^{-1} \cdot \mathrm{m}^{-1}$ arranged from the outer layer to that touching the internal conducting walls of the enclosure.

If the single aperture $\left(10 \times 10 \mathrm{~cm}^{2}\right)$ is split into four apertures (each $5 \times 5 \mathrm{~cm}^{2}$ ) arranged in two rows and two columns, the internal coating has more considerable improvement of the shielding effectiveness of the rectangular enclosure over a wide frequency band. This is clear in Figure 15, where the minimum value is about $20 \mathrm{~dB}$ over the entire frequency band of operation $(0-1 \mathrm{GHz})$. Even for higher frequencies, the internal coating in this case is still capable of preserving

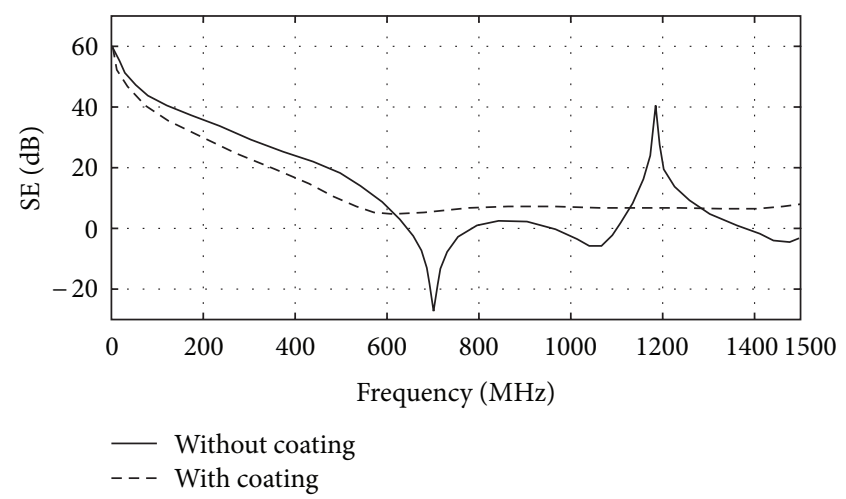

FIGURE 14: Shielding effectiveness versus frequency of a $30 \times$ $12 \times 30 \mathrm{~cm}^{3}$ box-shaped enclosure with a $10 \times 10 \mathrm{~cm}^{2}$ aperture illuminated by a plane wave at normal incidence in the presence and absence of a lossy internal coating of $1.5 \mathrm{~cm}$ thickness.

the shielding effectiveness of the rectangular enclosure above $20 \mathrm{~dB}$.

4.2.4. Improvement of the Shielding Effectiveness of Open Enclosures at Internal Resonance Using External Spatial Filter. The severe degradation of the shielding effectiveness of the open enclosure occurs in narrow band intervals around the frequencies corresponding to the internal resonances of the enclosure cavity. If a band-stop spatial filter [15] is placed outside the shield in front of the aperture such that most of the incident power at a cavity resonant frequency is filtered out before penetrating the cavity, this will considerably improve its shielding effectiveness at this frequency. Of course the, spatial filter will be designed so that its stop band is centered on the resonant frequency of the shield cavity. According to previous discussions, the worst value of the shielding effectiveness of a rectangular enclosure of the dimensions $30 \times 12 \times 30 \mathrm{~cm}$ with aperture of dimensions $10 \times 10 \mathrm{~cm}$ is obtained at about $0.7 \mathrm{GHz}$. A band-stop filter can be constructed up as a planner array of conducting strips as shown in Figure 16. The frequency response of this filter is shown in Figure 17. As shown in this figure, the stop band of this filter is centered on $0.78 \mathrm{GHz}$.

Figure 18 shows the variation of the shielding effectiveness of an open enclosure with a spatial filter screen placed in front of its aperture, as shown in Figure 16 over the operating frequency band compared with that of the same enclosure without the spatial filter screen. It is clear that the shielding effectiveness is improved at the resonant frequency and over the higher frequency band such that it is almost kept greater than $0 \mathrm{~dB}$ over the entire frequency range $(0-1.0 \mathrm{GHz})$.

\subsubsection{Improvement of Shielding Effectiveness of Open Enclo-} sures by Combination of Multiple Methods. We can combine more than one technique of those previously discussed for further improvement of the shielding effectiveness of open enclosures. Figure 19 shows the effect of using a lossy coating and, at the same time, placing a spatial filter screen in front of the aperture of an open rectangular metallic enclosure on 


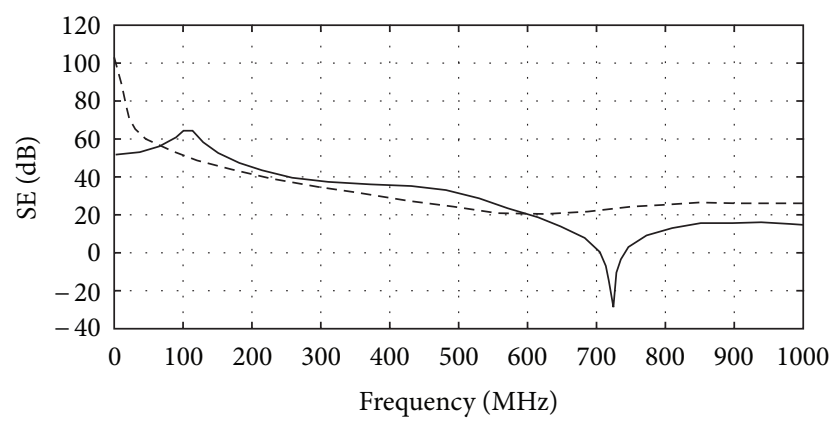

Four $5 \mathrm{~cm} \times 5 \mathrm{~cm}$ apertures without coating

- - Four $5 \mathrm{~cm} \times 5 \mathrm{~cm}$ apertures with internal coating

FIGURE 15: Shielding effectiveness versus frequency of a $30 \times 12 \times$ $30 \mathrm{~cm}^{3}$ box-shaped enclosure with four $5 \times 5 \mathrm{~cm}^{2}$ apertures arranged in two rows and two columns, illuminated by a plane wave at normal incidence in the presence and absence of a lossy internal coating of $1.5 \mathrm{~cm}$ thickness.

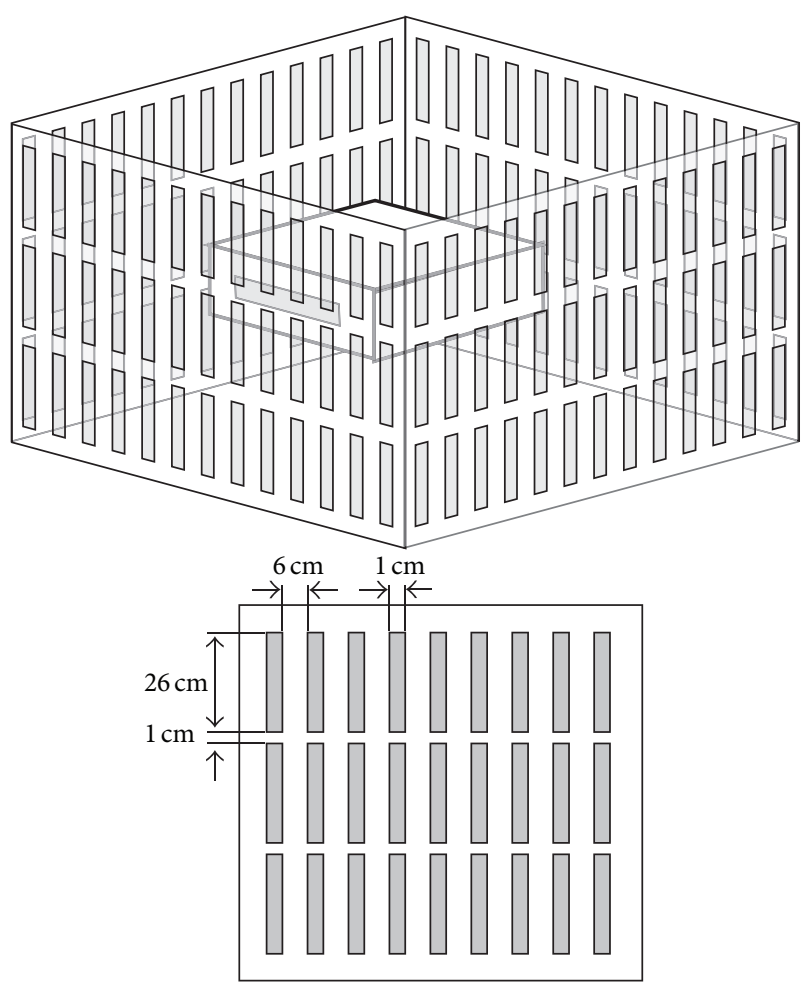

FIGURE 16: Housing the open enclosures in frequency selective walls.

the shielding effectiveness over the operating frequency range compared with that of the original enclosure. It is obvious that this configuration gives better shielding at the resonant frequency, considering a $30 \times 12 \times 30 \mathrm{~cm}$ enclosure with $10 \times$ $10 \mathrm{~cm}$ aperture, than that obtained using the internal coating or the filter screen alone. Figure 20 shows a comprehensive comparison for some possible combinations of shielding improvement techniques.

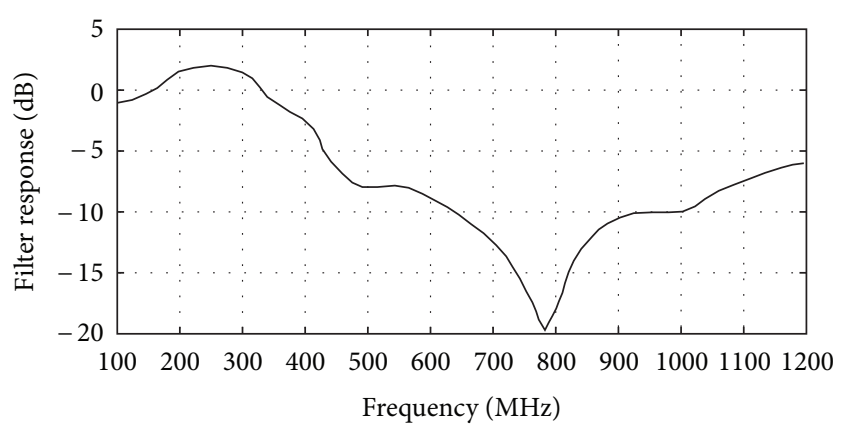

FIGURE 17: Frequency response of the band-stop filter composed of planner array of strips.

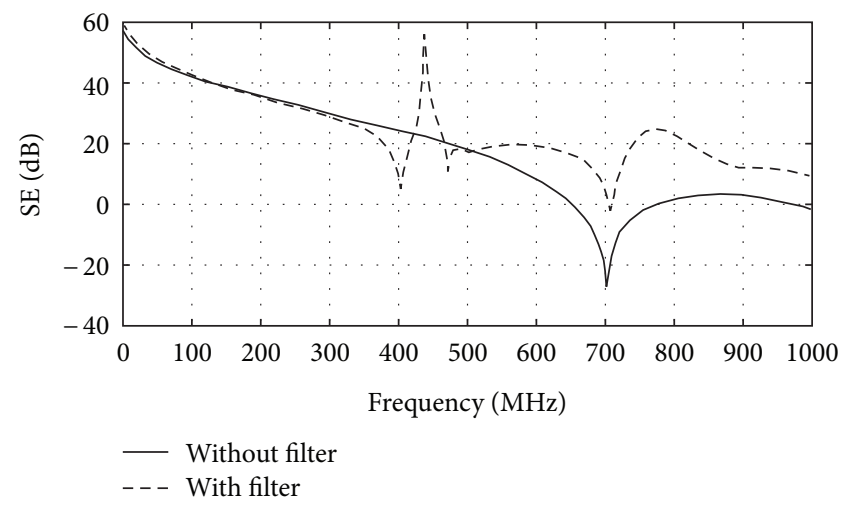

FIGURE 18: Shielding effectiveness versus frequency for a $30 \times 12 \times$ $30 \mathrm{~cm}^{3}$ enclosure with a $10 \times 10 \mathrm{~cm}^{2}$ aperture illuminated by a plane wave at normal incidence with a spatial filter of $3 \times 5$ conducting strips, placed $4 \mathrm{~cm}$ away from the aperture.

Now, let us summarize the methods presented throughout the previous discussions to improve the shielding effectiveness of rectangular metallic enclosures with rectangular apertures; these include (i) increasing aspect ratio of the rectangular aperture with keeping its area, (ii) splitting the apertures into a larger number of apertures with keeping the same opening area, (iii) coating the internal walls of the metallic enclosure with a multilayered lossy material with the appropriate conductivity profile, and, finally, (iv) placing an external electromagnetic filter screen at the proper distance in front of the aperture of the metallic enclosure. If all these methods are used together to get a combined solution, the resulting shielding effectiveness, as a function of the frequency, will be like that shown in Figure 21 compared to that of the original enclosure. It is clear that this solution is the best for a $30 \times 12 \times 30 \mathrm{~cm}$ enclosure with a $10 \times$ $10 \mathrm{~cm}$ aperture as regarding the shielding effectiveness over the entire frequency range and at the internal resonances as well.

\section{Conclusion}

The characteristics of the electromagnetic shielding effectiveness of a metallic enclosure with a rectangular aperture 


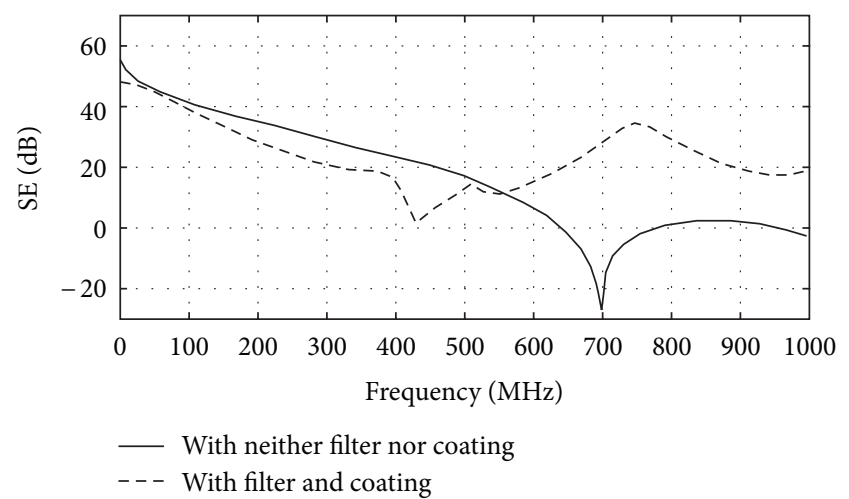

FIGURE 19: Shielding effectiveness versus frequency for an internally coated $30 \times 12 \times 30 \mathrm{~cm}^{3}$ enclosure with a $10 \times 10 \mathrm{~cm}^{2}$ aperture illuminated by a plane wave at normal incidence with a spatial filter of $3 \times 5$ conducting strips, placed $4 \mathrm{~cm}$ away from the aperture.

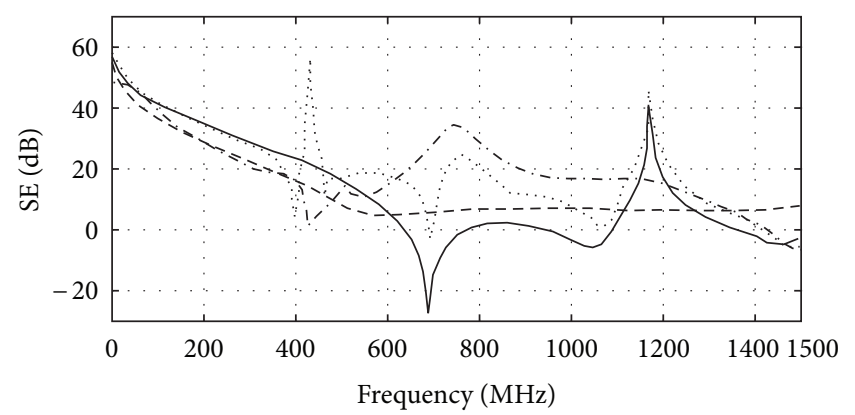

$$
\begin{array}{ccc}
\text { _ With neither filter nor coating } & \ldots . . . & \text { With filter only } \\
\text { - - - With internal coating only } & -. \cdot- & \text { With both filter } \\
& \text { and coating }
\end{array}
$$

FIGURE 20: Shielding effectiveness versus frequency at the center of a $30 \times 12 \times 30 \mathrm{~cm}^{3}$ enclosure with a $10 \times 10 \mathrm{~cm}^{2}$ aperture illuminated by a plane wave at normal incidence.

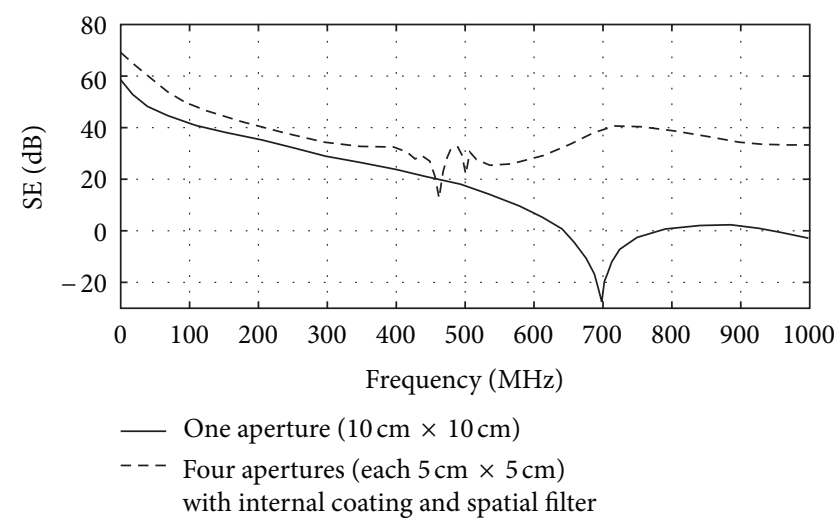

FIGURE 21: Shielding effectiveness versus frequency of a $30 \times 12 \times$ $30 \mathrm{~cm}^{3}$ enclosure with a single $10 \times 10 \mathrm{~cm}^{2}$ aperture and without internal coating and without a spatial filter compared to that of an internally coated enclosure with four $5 \times 5 \mathrm{~cm}^{2}$ apertures and with a spatial filter of $3 \times 5$ conducting strips, placed $4 \mathrm{~cm}$ away from the apertures when both are illuminated by a plane wave at normal incidence. are studied over a wide range of the frequency. It is shown that the shielding effectiveness is severely degraded at the internal resonances of the enclosure cavity and may decrease below $-40 \mathrm{~dB}$. A variety of methods are suggested to improve the shielding effectiveness of rectangular enclosures with rectangular apertures over a wide frequency range and at the internal resonances of the metallic enclosure. The suggested methods include increasing aspect ratio of the rectangular aperture with keeping its area, splitting the opening into a number of apertures with keeping the same opening area, coating the internal walls of the metallic enclosure with a multilayered lossy material of the appropriate conductivity profile, and, finally, placing an external electromagnetic band-stop frequency selective screen at the proper distance in front of the aperture of the metallic enclosure. It is shown that any of these methods when applied alone lead to a significant improvement of the shielding effectiveness. Also it is shown that various combinations of more than one method of those suggested in the present work result in further improvements of the shielding effectiveness. A combination of all these methods (applied together) leads to a minimum shielding effectiveness of $20 \mathrm{~dB}$ over the operating frequency band. The results concerning the effect of the angle of incidence and polarization of the incident plane on the shielding effectiveness of the enclosure over the entire frequency range are presented.

\section{References}

[1] P. Dehkhoda, A. Tavakoli, and R. Moini, "Fast calculation of the shielding effectiveness for a rectangular enclosure of finite wall thickness and with numerous small apertures," Progress in Electromagnetics Research, vol. 86, pp. 341-355, 2008.

[2] P. V. Y. Jayasree, V. S. S. N. Srinivasa Baba, B. Prabhakara Rao, and P. Lakshman, "Analysis of shielding effectiveness of single, double and laminated shields for oblique incidence of EM waves," Progress in Electromagnetics Research B, no. 22, pp. 187-202, 2010.

[3] C. D. Raj, G. S. Rao, P. V. Y. Jayasree, B. Srinu, and P. Lakshman, "Estimation of reflectivity and shielding effectiveness of three layered laminate electromagnetic shield at X-band," Progress in Electromagnetics Research B, no. 20, pp. 205-223, 2010.

[4] Z. A. Khan, C. F. Bunting, and M. D. Deshpande, "Shielding effectiveness of metallic enclosures at oblique and arbitrary polarizations," IEEE Transactions on Electromagnetic Compatibility, vol. 47, no. 1, pp. 112-122, 2005.

[5] V. Rajamani, C. F. Bunting, M. D. Deshpande, and Z. A. Khan, "Validation of Modal/MoM in shielding effectiveness studies of rectangular enclosures with apertures," IEEE Transactions on Electromagnetic Compatibility, vol. 48, no. 2, pp. 348-353, 2006.

[6] K. F. A. Hussein, "Effect of internal resonance on the radar cross section and shield effectiveness of open spherical enclosures," Progress in Electromagnetics Research, vol. 70, pp. 225-246, 2007.

[7] B. W. Kim, Y. C. Chung, and T. K. Kang, "Analysis of electromagnetic penetration through apertures of shielded enclosure using finite element method," in Proceedings of the 14th Annual Review of Progress in Applied Computational Electromagnetics, vol. 2, pp. 795-798, Monterey, Calif, USA, March 1998. 
[8] J. Z. Lei, C. H. Liang, and Y. Zhang, "Study on shielding effectiveness of metallic cavities with apertures by combining parallel FDTD method with windowing technique," Progress in Electromagnetics Research, vol. 74, pp. 85-112, 2007.

[9] J. Z. Lei, C. H. Liang, and Y. Zhang, "Study on shielding effectiveness of metallic cavities with apertures by combining parallel FDTD method with windowing technique," Progress in Electromagnetics Research, vol. 74, pp. 85-112, 2007.

[10] K. S. Yee, "Numerical solution of intial boundary value problems involving Maxwell's equations in isotropic media," IEEE Transactions on Antennas and Propagation, vol. 14, no. 3, pp. 302-307, 1966.

[11] A. Taflove and S. Hagness, Computational Electrodynamics: The Finite-Difference Time-Domain Method, Artech House, Boston, Mass, USA, 2000.

[12] A. Taflove and M. E. Brodwin, "Numerical solution of steady state electromagnetic scattering problems using the time dependent Maxwell's equations," IEEE Transactions on Microwave Theory and Techniques, vol. 23, no. 8, pp. 623-630, 1975.

[13] J. P. Berenger, "A perfectly matched layer for the absorption of electromagnetic waves," Journal of Computational Physics, vol. 114, no. 2, pp. 185-200, 1994.

[14] E. S. Siah, K. Sertel, J. L. Volakis, V. V. Liepa, and R. Wiese, "Coupling studies and shielding techniques for electromagnetic penetration through apertures on complex cavities and vehicular platforms," IEEE Transactions on Electromagnetic Compatibility, vol. 45, no. 2, pp. 245-257, 2003.

[15] A. E. Farahat, K. F. A. Hussein, and N. M. El-Minyawi, "Spatial filters for linearly polarized antennas using free standing frequency selective surface," Progress in Electromagnetics Research M, vol. 2, pp. 167-188, 2008. 

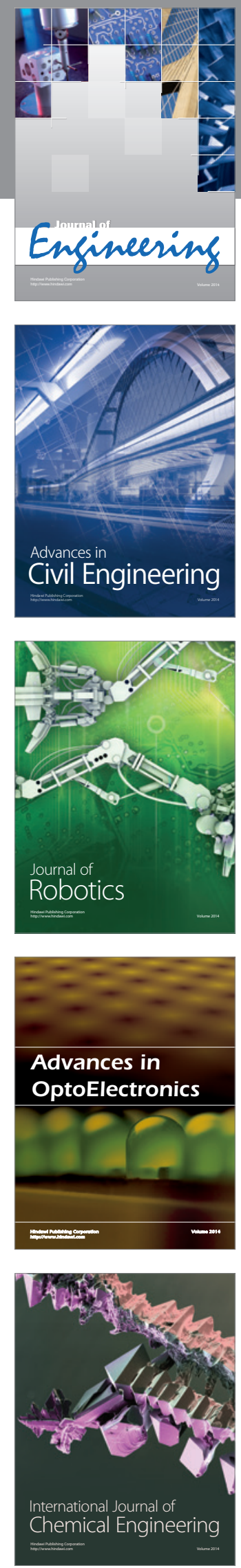

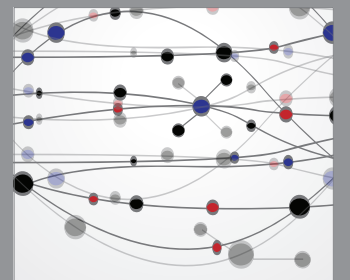

The Scientific World Journal
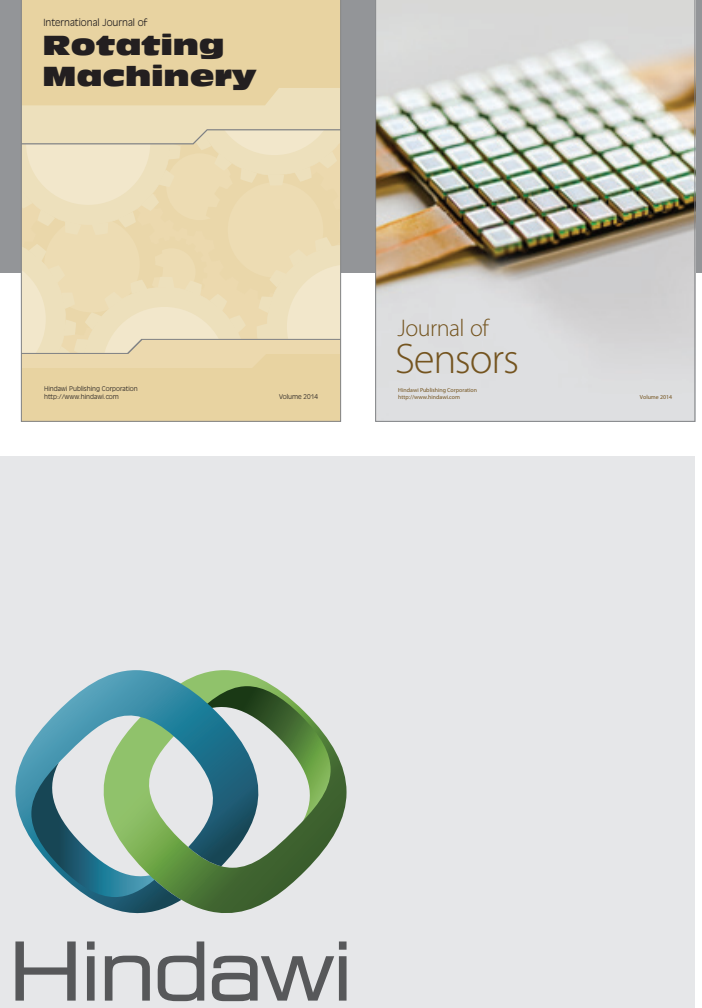

Submit your manuscripts at http://www.hindawi.com
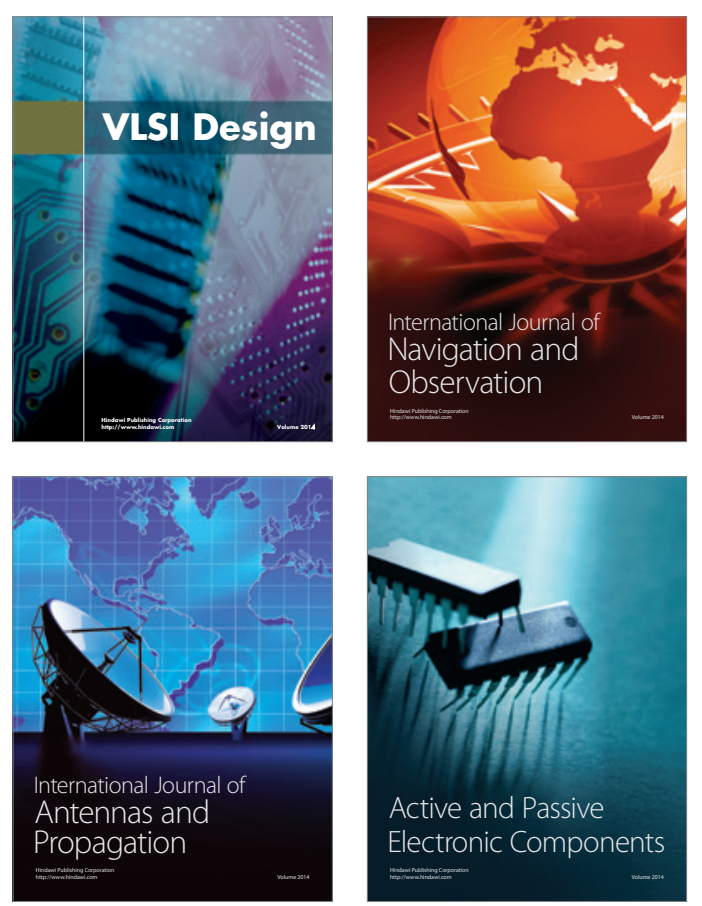
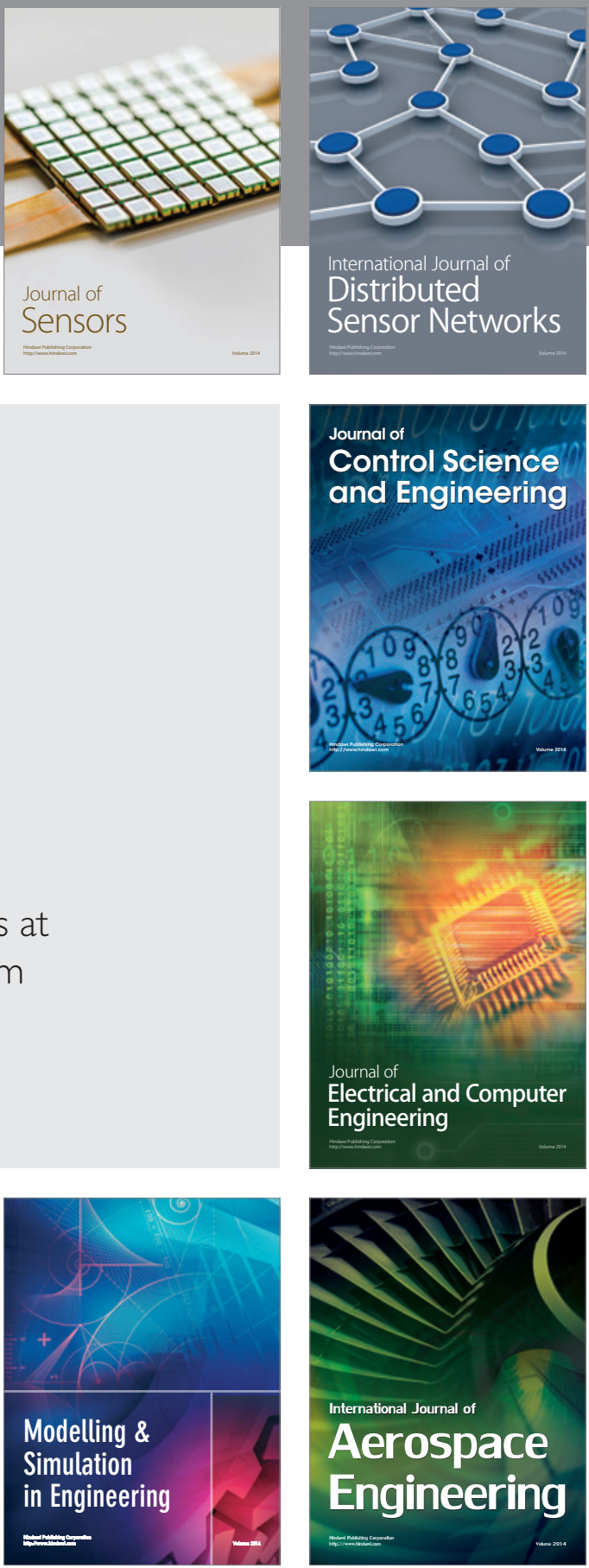

Journal of

Control Science

and Engineering
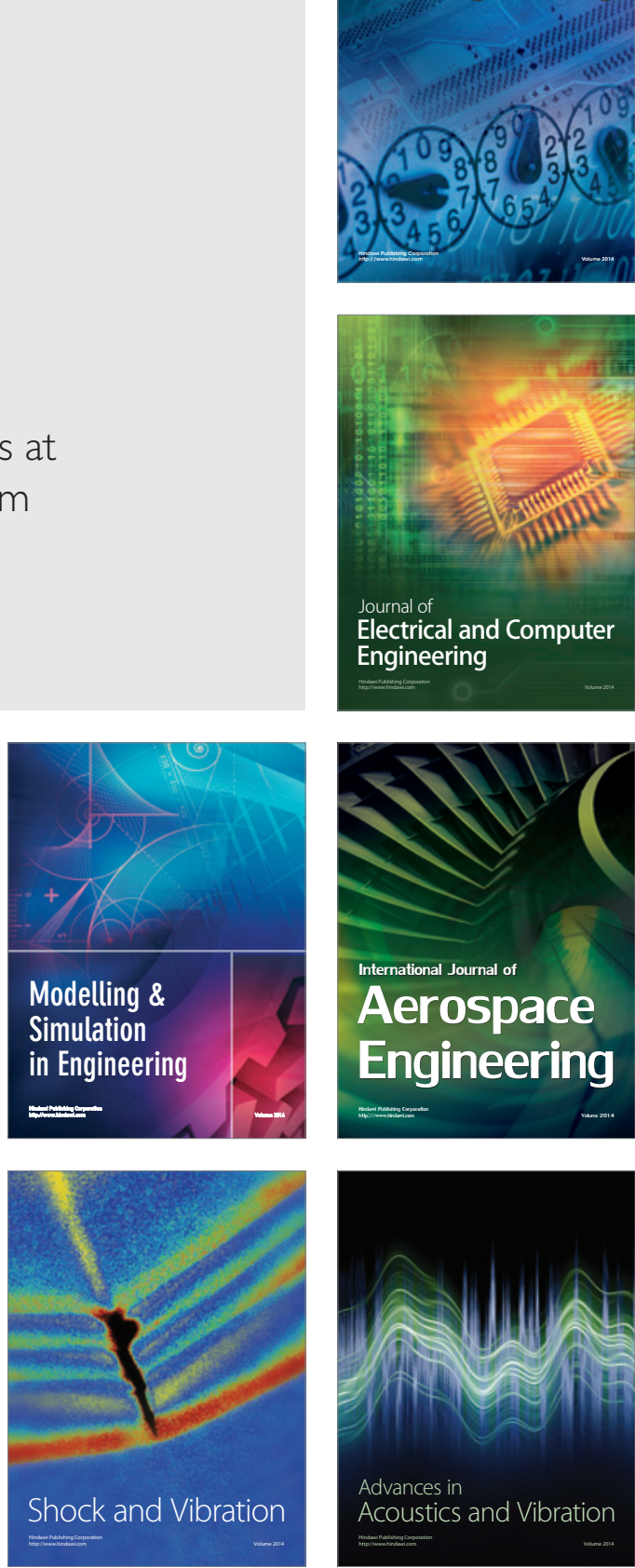